\title{
Mapping of three QTLs that regulate internode elongation in deepwater rice
}

\author{
Yoko Hattori ${ }^{+1,2)}$, Keisuke Nagai ${ }^{+1)}$, Hitoshi Mori ${ }^{3)}$, Hidemi Kitano'), Makoto Matsuoka ${ }^{1)}$ \\ and Motoyuki Ashikari*1) \\ 1) Bioscience and Biotechnology Center, Nagoya University, Furo-cho, Chikusa, Nagoya, Aichi 464-8601, Japan \\ 2) Japan Society for the Promotion of Science, Ichibancho 8, Chiyoda, Tokyo 102-8472, Japan \\ 3) Graduate School of Bioagricultural Sciences, Nagoya University, Furo-cho, Chikusa, Nagoya, Aichi 464-8601, Japan
}

The internodes of deepwater rice can elongate in response to rises in water level. This unique character allows deepwater rice to survive severe flooding during the monsoon season in South and Southeast Asia. Our previous quantitative trait locus (QTL) analysis of a deepwater rice cultivar (Oryza sativa) detected QTLs on chromosomes 1, 3 and 12. In this study, we produced three nearly isogenic lines (NILs) possessing each of the three QTLs by backcross introduction of each chromosomal region into a non-deepwater rice cultivar. The NILs showed internode elongation under deepwater conditions, and we were able to demonstrate the existence of the QTLs and to evaluate the effect of each QTL. Using progenies of the NILs, we mapped all QTLs between molecular markers. Comparison of the location of the most effective QTL between the rice cultivar (O. sativa) and a wild rice species (O. rufipogon) indicated that the QTL on chromosome 12 is common and is the most important QTL for internode elongation in deepwater condition.

Key Words: rice, QTL, deepwater rice, internode elongation.

\section{Introduction}

The most remarkable characteristic of deepwater rice is rapid internode elongation when subjected to deep water. Deepwater rice does not show significant internode elongation in shallow water, whereas deep water induces dramatic internode elongation. Most ordinary rice cultivars planted in irrigated fields do not have this characteristic. Deepwater rice is mainly cultivated in lowland areas of South and Southeast Asia that are flooded during the rainy season. Rapid internode elongation in response to deep water in deepwater rice is necessary to avoid anoxia. Deepwater rice elongates internodes to keep the top leaves above the water surface to allow gas exchange. Remarkable internode growth occurs during flooding, with daily increases in plant height of 20 to $25 \mathrm{~cm}$; ultimately, plants can reach a height of up to $7 \mathrm{~m}$ (Vergara et al. 1976, Catling 1992).

This rapid growth in deep water of deepwater rice is unique and may provide insights into unknown mechanisms of plant growth regulation. Physiological studies have proposed that the plant hormones ethylene, ABA, and gibberellin are involved in deepwater rice elongation (for review, see Kende et al. 1998). Deepwater characteristics have been reported to be controlled by duplicate genes (efl and ef2; Ramiah and Ramaswami 1940), a partially dominant gene (Hamamura and Kupkanchankul 1979), a single dominant

Communicated by M. Yano

Received October 22, 2007. Accepted December 13, 2007.

*Corresponding author (e-mail: ashi@agr.nagoya-u.ac.jp)

$\dagger$ These authors contributed equally to this work gene (Tripathi and Balakrishna Rao 1985), an incomplete dominant gene (Suge 1987) and a single recessive gene (dw3; Eiguchi et al. 1993). Despite these reports, however, the genes that regulate internode elongation under deepwater conditions have not been identified or mapped.

Many agronomic traits are governed by sets of genes known as quantitative trait loci (QTLs; Yano 2001, Yano and Sasaki 1997, Ashikari and Matsuoka 2006). Since QTL analysis can detect genes of complex traits, it might be suitable to detect genes that regulate rapid internode elongation in deepwater rice. For QTL analysis, it is necessary to determine methods to measure target traits. To evaluate deepwater characteristics, Inouye (1983) proposed using the position of the lowest elongated internode (LEI). Nemoto et al. (2004) first applied LEI in diallel and QTL analysis of deepwater characteristics and detected two QTLs on chromosomes 3 and 12. Vergara and Mazaredo (1979) proposed measuring total internode elongation to evaluate deepwater characteristics. We previously employed both LEI and total internode elongation to evaluate deepwater rice characteristics (Hattori et al. 2007). In addition to measuring LEI, we measured total internode elongation length (TIL) and the number of elongated internodes (NEI) to evaluate total internode elongation. Using these three parameters, we performed QTL analysis with an $\mathrm{F}_{2}$ population from a cross between a deepwater rice cultivar, C9285 (Oryza sativa ssp. indica), and a non-deepwater rice cultivar, Taichung 65 (T65, O. sativa ssp. japonica). We subsequently detected five QTLs: $q$ TIL1 $1^{\text {C9285 }}$ on chromosome 1, qLEI3 ${ }^{C 9285}$ on chromosome 3 and qTIL12C9285, qNEI12C9285 and $q L E I 12^{C 9285}$ on chromosome 12 (Hattori et al. 2007). 
To understand the molecular mechanism of deepwater characteristics it is necessary to clone the QTLs. Initial step of cloning the QTLs, we have to confirm their existence, and then to evaluate them. The detected QTLs in cultivated deepwater rice have not yet been verified. In this paper, we developed nearly isogenic lines (NILs), used them to demonstrate the existence of the QTLs and evaluated the effects of each QTL. Furthermore, using progenies of heterozygotes of each NIL, we mapped the QTLs. Among the detected QTLs, we then compared the location of the most effective QTLs in cultivated deepwater rice and in the wild rice species $O$. rufipogon, which has deepwater characteristics.

\section{Materials and Methods}

\section{Plant materials}

Two kinds of deepwater rice were used for this study: a deepwater rice cultivated in Bangladesh, C9285 (Oryza sativa ssp. indica), and a wild rice species, W0120 (O. rufipogon; perennial type), from India that has deepwater characteristics (Morishima et al. 1962). These two deepwater rice varieties were provided by the National Institute of Genetics in Japan (http://www.shigen.nig.ac.jp/rice/ oryzabase/top/top.jsp). The non-deepwater rice cultivar, Taichung 65 (T65; O. sativa ssp. japonica), was used as a control and also as crossing material in this study. T65 is maintained at Nagoya University.

\section{Producing NILs}

To produce NILs harboring the target QTL regions from C9285 in a T65 background, $\mathrm{F}_{1}$ plants (T65/C9285) were backcrossed four times with T65 using marker-assisted selection (Yano and Sasaki 1997, Yano 2001). Since our previous study (Hattori et al. 2007) detected QTLs at three positions, we developed three NILs, NIL-1 ${ }^{\text {C9285 }}$, NIL-3 ${ }^{\text {C9285 }}$ and NIL-12 ${ }^{\mathrm{C} 9285}$ in this study. These NILs were selected from the $\mathrm{BC}_{4} \mathrm{~F}_{2}$ generation.

\section{Phenotypic evaluation}

To evaluate elongation ability in deepwater rice, we selected three parameters: total internode elongation length (TIL), number of elongated internode (NEI) and lowest elongated internode (LEI) position on the main tiller. Plants were germinated in Petri dishes in water at $30^{\circ} \mathrm{C}$ for $72 \mathrm{~h}$ and then transplanted into pots $(10 \mathrm{~cm}$ diameter, $12 \mathrm{~cm}$ height $)$. The plants were grown in air (i.e., approximately $5 \mathrm{~cm}$ of water) and at the ten-leaf stage, they were submerged in water up to $70 \%$ of the plant height in 3,000-L tanks for 1 week prior to determining the TIL and NEI.

To determine the LEI position, seeds were pregerminated $\left(30^{\circ} \mathrm{C}, 72 \mathrm{~h}\right)$ and transplanted to plug plates (each plug measured $125 \mathrm{~cm}^{3}$ ). At the 12-leaf stage, the plants were immersed in a deepwater tank up to about $70 \%$ of the plant height for 10 days. The position of the lowest elongated internode longer than $5 \mathrm{~mm}$ was defined as the LEI position and determined (Inouye 1987).

\section{Determination of the QTL positions}

DNA was extracted from individuals using the TPS method (Hattori et al. 2007) and was used for genotyping with molecular markers. PCR-based markers, including simple-sequence repeat markers (McCouch et al. 2002, Ware et al. 2002, http://www.gramene.org/) and cleaved amplified polymorphic sequence markers (Konieczny and Ausubel 1993), or single nucleotide polymorphisms (SNPs) were selected by comparing the genomic sequence of each parent and were applied for linkage analysis. PCR was performed as described by Chen et al. (1997). The amplified products from each population were separated on $3 \%$ agarose gels in $0.5 \times$ TBE buffer and visualized using ethidium bromide. Map positions of the QTLs were determined by comparison between the genotypes and phenotypes (Detail procedures are described in results).

\section{Results}

\section{Constructing NILs}

We previously reported five QTLs: $q T I L 1^{C 9285}$ on chromosome 1, qLEI3 ${ }^{C 9285}$ on chromosome 3, and $q$ TIL12C9285, $q N E I 12^{C 9285}$ and $q L E I 12^{C 9285}$ on chromosome 12 (Fig. 1A); all C9285 (deepwater cultivar) alleles enhanced internode elongation (Hattori et al. 2007). For QTL cloning, it is necessary to demonstrate the existence of the QTLs using NILs and to evaluate the effect of the QTLs. We produced NILs having the QTLs of the C9285 allele in a T65 (nondeepwater rice) genetic background. First, T65 was backcrossed to $F_{1}$ plants (T65/C9285) four times. During the backcrossing, we selected lines with marker-assisted selection that had the target QTL region of C9285, and these lines were further crossed. In the $\mathrm{BC}_{4} \mathrm{~F}_{2}$ generation, we selected lines carrying the $\mathrm{C} 9285$ chromosome segment around the target QTLs in the T65 genetic background. We produced three NILs: NIL-1 ${ }^{\mathrm{C} 9285}$ possesses $q$ TIL $1^{\text {C9285 }}$, NIL-3 ${ }^{\mathrm{C} 9285}$ possesses $q L E I 3^{C 9285}$ and NIL-12 ${ }^{\mathrm{C} 9285}$ possesses $q T I L 12^{\mathrm{C} 9285}$, $q N E I 12^{C 9285}$ and $q L E I 12^{C 9285}$ (Fig. 1B-D).

The molecular marker RM246 on chromosome 1 showed the T65 genotype, and molecular markers RM1183, RM7180 and RM6840 showed the C9285 genotype in NIL$1^{\mathrm{C} 9285}$ (Fig. 1B-i, ii and iii). Other molecular markers covering all chromosomes indicated T65 genotypes (data not shown). We concluded that a line possessing at least the chromosome fragment between RM1183 and RM6840 of chromosome 1 from $\mathrm{C} 9285$ in a T65 genetic background can be considered a NIL of $q$ TIL $1^{\text {C9285 }}$ and named it NIL-1 ${ }^{\text {C9285 }}$ (Fig. 1B-i). Similarly, molecular markers RM232 and RM156 on chromosome 3 had a T65 genotype, and molecular markers RM3803 and RM7249 on chromosome 3 had the C9285 genotype in the candidate line of NIL for $q L E I 3^{C 9285}$ (Fig. 1C-i, ii and iii). Other molecular markers covering all chromosomes had T65 genotypes (data not shown). A line possessing at least the chromosome 3 region between RM3803 and RM7249 from C9285 in a T65 genetic background was considered to be a NIL of $q L E I 3^{C 9285}$ and was 
named NIL-3 ${ }^{\mathrm{C} 9285}$ (Fig. 1C-i). For selection of NILs possessing QTLs on chromosome 12, we selected a line with the molecular marker G2140 on chromosome 12 with the T65 genotype, and molecular markers RM5479, RM6386, and RM235 on chromosome 12 with the C9285 genotype (Fig. 1D-i, ii and iii). Other molecular markers covering the chromosome indicated T65 genotypes (data not shown). The line possessing at least the genomic fragment between RM5479 and RM235 from C9285 on chromosome 12 in a T65 genetic background was considered a NIL for qTIL12 $2^{\text {C9285 }}, q N E I 12^{\text {C9285 }}$ and $q L E I 12^{\text {C9285 }}$ and was named NIL-12 ${ }^{\mathrm{C} 9285}$.

\section{Evaluation of QTL effects using NILs}

Since NILs carrying only one target QTL region in a unique other genome background can eliminate the effects of other QTLs, such material is useful to demonstrate the existence of a QTL and to evaluate the effect of the target QTL. We investigated the existence of each QTL of the C9285 allele using NIL-1 ${ }^{\mathrm{C} 9285}$, NIL-3 ${ }^{\mathrm{C} 9285}$ and NIL-12C9285 (Fig. 1B-
D). Each NIL was submerged in deep water for 1 week, and three parameters (TIL, NEI and LEI) were observed. In the air, average TILs were $0 \mathrm{~cm}$ in NIL- $1^{\text {C9285 }}, 0 \mathrm{~cm}$ in NIL-3 $3^{\mathrm{C} 9285}$ and $0.1 \mathrm{~cm}$ in NIL-12 ${ }^{\mathrm{C} 9285}$; however, in deepwater conditions, internode lengths were $9.8 \mathrm{~cm}$ in NIL- $1^{\mathrm{C} 9285}, 3.6 \mathrm{~cm}$ in NIL-3 $3^{\mathrm{C} 9285}$ and $17.6 \mathrm{~cm}$ in NIL-12 ${ }^{\mathrm{C} 9285}$ (Fig. 2A). The average NEI was 0 in NIL-1 ${ }^{\mathrm{C} 9285}, 0$ in NIL-3 ${ }^{\mathrm{C} 9285}$ and 0.1 in NIL$12^{\mathrm{C} 9285}$ in air; but in water these increased to 1.1 in NIL$1^{\mathrm{C} 9285}, 0.4$ in NIL-3 ${ }^{\mathrm{C} 9285}$ and 2.0 in NIL-12 ${ }^{\mathrm{C} 9285}$ (Fig. 2B). The control plant, $\mathrm{T} 65$, showed little response to deep water, with a TIL of $0 \mathrm{~cm}$ in air and $0.8 \mathrm{~cm}$ in deep water, and an NEI of 0 in air and $0.2 \mathrm{~cm}$ in deep water.

The LEI scores were $10-12$ in NIL-1 ${ }^{\text {C9285 }}, 11-12$ in NIL-3 $3^{\mathrm{C} 9285}$ and $9-10$ in NIL-12C9285 (Fig. 2C). The LEI in T65 could not be evaluated because it showed little response to water level. Comparisons of the response to water of the three traits between the NILs and T65 indicated that the three NILs possess QTLs from C9285 that can respond to water level and enhance internode elongation in deep water.
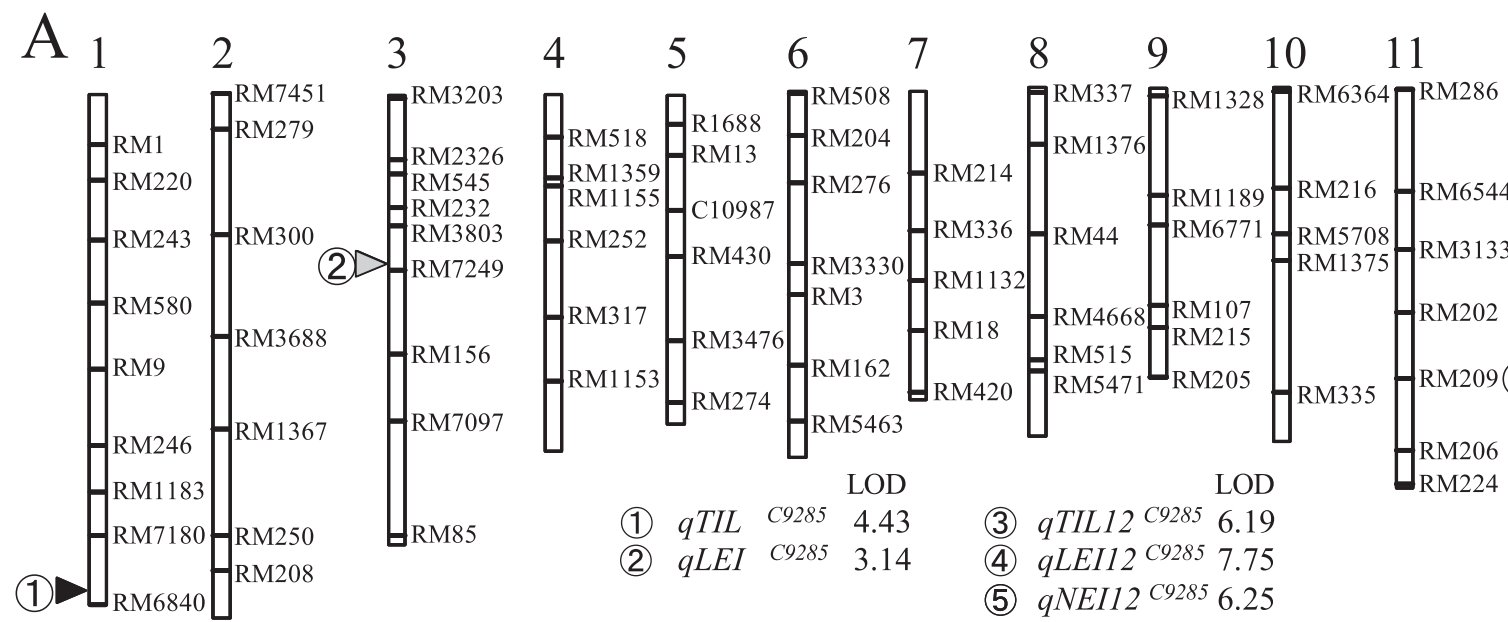

12

B

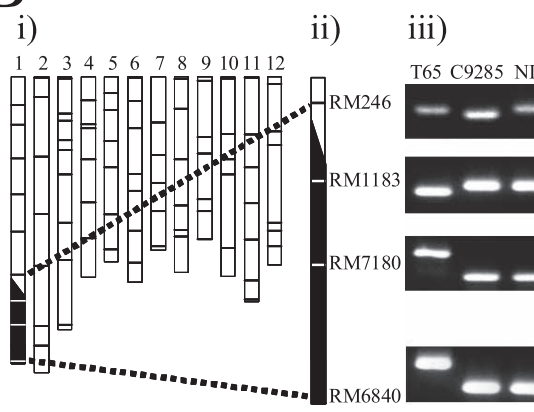

C

i)

(3) $q$ TIL12 ${ }^{C 9285} 6.19$

(4) $q L E I 12 C 92857.75$

(5) $q N E I 12^{C 9285} 6.25$

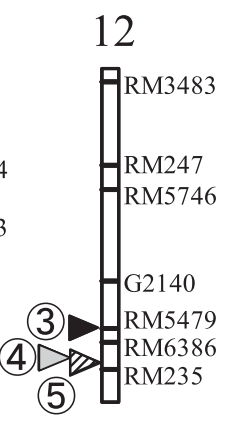

$\prod 20 \mathrm{cM}$

\section{$\mathrm{D}$}
i)

ii) iii)

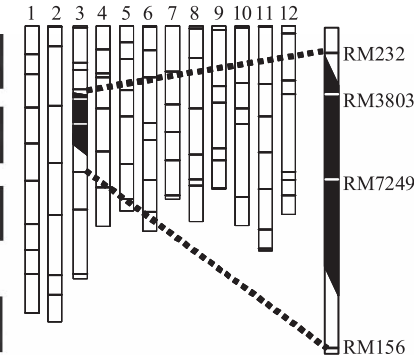

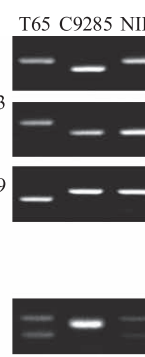

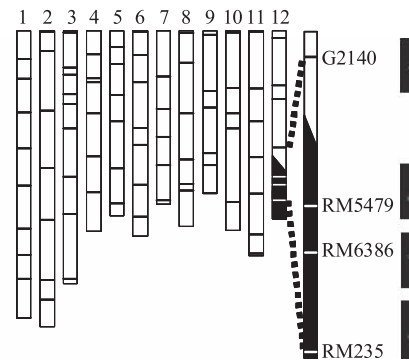

iii)

T65 C9285 NIL12
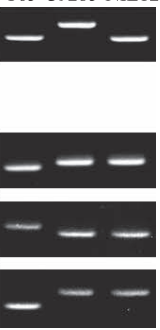

Fig. 1. Location of QTLs and graphical genotypes of nearly isogenic lines (NILs). (A) Position of the QTL for deepwater characteristics on the rice chromosome. QTL positions are illustrated based on results of QTL analysis using the deepwater rice cultivar C9285 (Hattori et al. 2007). The five detected QTL positions are indicated as 1-5. Arrowheads indicate QTL peaks. QTL names and LOD scores are indicated under the

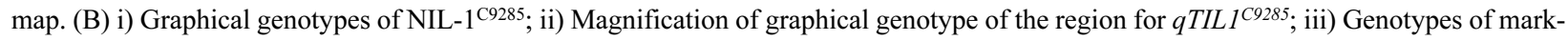

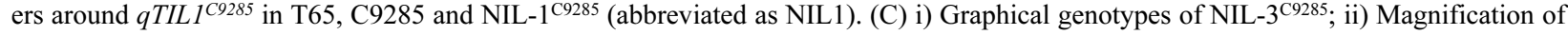
the graphical genotype of region for $q L E I 3^{C 9285}$; iii) Genotypes of markers around $q L E I 3^{C 9285}$ in T65, C9285 and NIL-3 ${ }^{\mathrm{C} 9285}$ (abbreviated as NIL3). (D) i) Graphical genotypes of NIL-12 ${ }^{\mathrm{C} 9285}$; ii) Magnification of graphical genotype of region for $q$ TIL12 ${ }^{\mathrm{C} 9285}$, $q$ NEI12 $2^{\mathrm{C} 2285}$ and qLEI12 ${ }^{\mathrm{C} 9285}$; iii) Genotypes of markers around $q$ TIL12 ${ }^{\mathrm{C} 9285}, q N E I 12^{\mathrm{C} 9285}$ and $q$ LEI12 ${ }^{\mathrm{C} 9285}$ in T65, C9285 and NIL-12 ${ }^{\mathrm{C} 9285}$ (abbreviated as NIL12). (B-D) T65 chromosome region is illustrated by a white box. C9285 chromosome region is illustrated by a black box. 

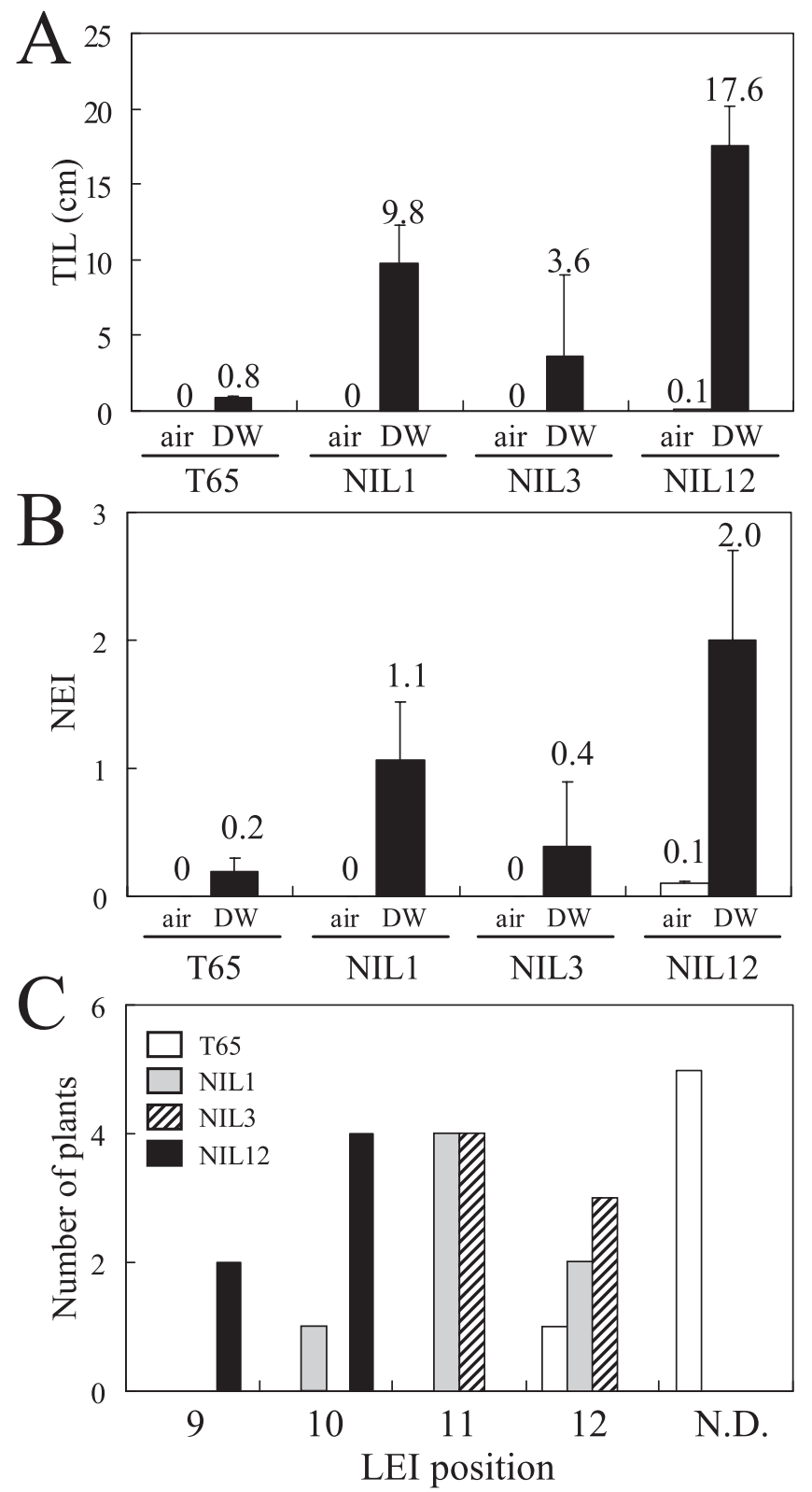

Fig. 2. Phenotype evaluation of NIL1, NIL3, and NIL12 plant response to deep water. (A) Quantitative internode elongation, total internode elongation length (TIL). (B) Quantitative internode elongation, number of elongated internodes (NEI). Values in a and $b$ are means with S.D. $(n=5)$. air, air conditions; DW, deepwater conditions. (C) Constitution of lowest elongated internode (LEI) position. Open bars indicate T65. Dotted bars indicate NIL$1^{\mathrm{C} 9285}$; hatched bars indicate NIL-3 ${ }^{\mathrm{C} 9285}$; solid bars indicate NIL$12^{\mathrm{C} 9285}$ (abbreviated as NIL1, NIL3 and NIL12, respectively).

\section{Mapping of QTLS}

Before constructing the linkage map, we compared the phenotypic differences among progenies with the NIL-1 ${ }^{\text {C9285 }}$ heterozygotes. The phenotypic difference in TIL between homozygous plants possessing the C9285 allele and homozygous plants having the T65 allele (Fig. 3A) was clearer than the phenotypic difference among homozygous plants possessing the C9285 or T65 alleles or plants heterozygous for their alleles (Fig. 3B). Accordingly, we used homozygotes to produce a reliable map.

To map qTIL1 ${ }^{C 9285}$, we first screened plants in which recombination occurred between RM246 and RM6840 from the 192 progenies of plants heterozygous for NIL-1 ${ }^{\mathrm{C} 9285}$ (Fig. 1B). Then, we selected 11 plants in which recombination occurred between RM246 and RM1183, 11 plants in which recombination occurred between RM1183 and RM7180, and 10 plants in which recombination occurred between RM7180 and RM6840. And then, we obtained several plants that were homozygous for the C9285 or T65 allele from the 32 progenies using molecular markers. These plants were submerged in deep water and their phenotypes were observed (data not shown). A comparison of the phenotypes and genotypes among the progenies of the 32 plants indicated that qTIL $^{\text {C9285 }}$ is located between RM7180 and RM6840 on chromosome 1 (Fig. 3C). To map qTIL1C9285 more precisely, ten plants in which recombination occurred between RM7180 and RM6840 were subjected to further mapping with the additional markers DWR1-B and DWR1S. For example, the recombination point of line no. 2 is between DWR1-B and DWR1-S and the phenotype does not show elongation (Fig. 3C), indicating that $q T I L 1^{C 9285}$ is located on the short-arm side of DWR1-S. In contrast, line no. 3 has the same recombination point between DWR1-B and DWR1-S, but this line had an elongated phenotype, and qTIL1 ${ }^{\text {C9285 }}$ is located on the long-arm side of DWR1-B. Hence, $q T I L 1^{C 9285}$ is located between DWR1-B and DWR1S. Comparison of the recombinant points and phenotypes in the other eight lines also indicated that $q T I L 1^{C 9285}$ is located between DWR1-B and DWR1-S (Fig. 3C).

Similarly, we first selected five plants each in which recombination occurred between RM232 and RM3803, between RM3803 and RM7249, and between RM7249 and RM156 from 192 progenies of plants heterozygous for NIL-

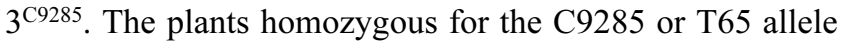
were selected from the progenies of these 15 recombinant plants; they were submerged and their phenotypes were determined. From the 15 recombinant lines, five plants that have recombination points between RM3803 and RM7249 are shown in Fig. 3d. A comparison of the phenotypes and recombination points indicated that $q L E I 3^{C 9285}$ is located between RM3803 and RM7249 (Fig. 3D).

Subsequently, we mapped qTIL12 $2^{\text {C9285 }}$, qNEI12 $2^{\mathrm{C} 9285}$, and $q L E I 12^{C 9285}$. Since TIL and NEI can be investigated in the same plant, we mapped $q T I L 12^{C 9285}$ and $q N E I 12^{C 9285}$ simultaneously. The genotypes of 192 progeny from heterozygous NIL-12 ${ }^{C 9285}$ plants were determined using the markers, and then the TIL and NEI phenotypes were observed. Similarly, we mapped qTIL12 $2^{C 9285}$ and $q N E I 12^{C 9285}$ to the same region between RM6386 and RM235 (Fig. 3E). Since scoring LEI differs from scoring TIL and NEI (see Materials and Methods), the same samples cannot be used. Therefore, another 96 progeny of NIL-12 ${ }^{C 9285}$ heterozygotes were scored for LEI, and $q L E I 12^{C 9285}$ was mapped between RM6386 and RM235 (Fig. 3F). 

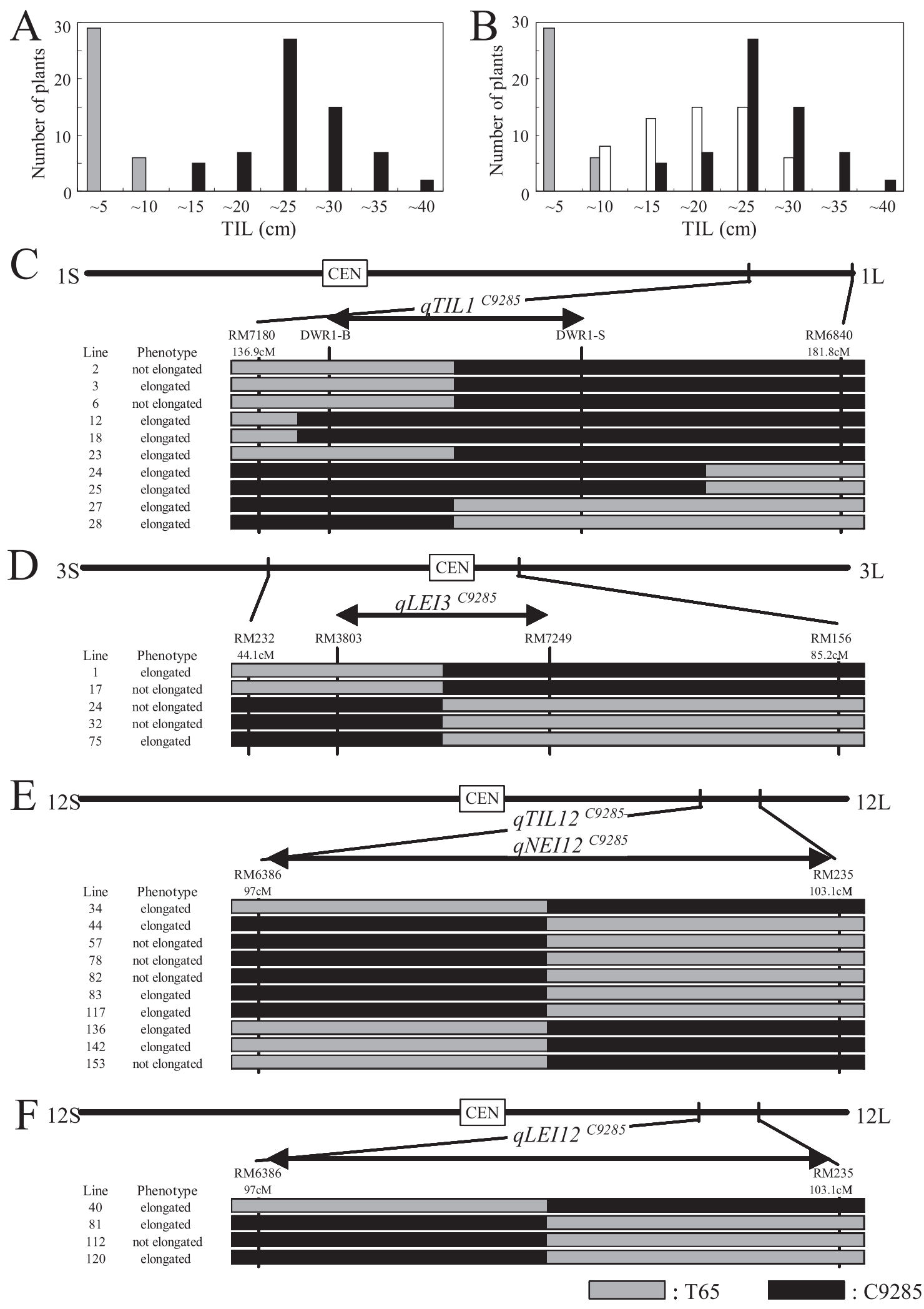

Fig. 3. Graphical genotype for mapping QTLs. (A) Phenotypic distribution of 2 genotypes (C9285 homozygote and T65 homozygote) derived from heterozygote of NIL-1 ${ }^{\text {C9285. }}$. (B) Phenotypic distribution of 3 genotypes (C9285 homozygote, T65 homozygote and their heterozygote) derived from heterozygote of NIL-1 ${ }^{\mathrm{C} 9285}$. (C) Mapping position of $q T I L 1^{C 9285}$. (D) Mapping position of $q L E I 3^{C 9285}$. (E) Mapping position of $q$ TIL12 $2^{C 9285}$ and $q N E I 12^{C 9285}$. (F) Mapping position of $q L E I 12^{C 9285}$. QTL regions are highlighted in the graphical genotype of recombinant lines with markers. Line name and phenotype are indicated to the left of each map. Gray bar indicates T65 chromosome. Black bar indicates C9285 chromosome. 
Integration of the map position of qTIL $12^{C 9285}$ and qTIL12 120120

The wild rice (O. rufipogon, W0120) shows deepwater characteristics (Morishima et al. 1962). QTL analysis using an $\mathrm{F}_{2}$ population derived from T65/W0120 detected three QTLs, qTIL12 $2^{\text {W0120 }}$, qNEI12 $2^{\text {W0120 }}$ and $q L E I 12^{\text {W0120 }}$, where the W0120 alleles enhanced internode elongation (Hattori et al. 2007). These three QTLs located in the same region on the long arm of chromosome 12 (Hattori et al. 2007). These positions are close to the location of $q T I L 12^{C 9285}$, $q N E I 12^{C 9285}$ and $q L E I 12^{C 9285}$ detected on chromosome 12 in C9285 (Fig. 1A). To investigate whether the QTLs in chromosome 12 in W0120 and the QTLs in C9285 are the same, we mapped $q T I L 12^{\text {W0120 }}$. Using the progeny of the heterozygote NIL-12 ${ }^{\mathrm{W} 0120}$ (Hattori et al. 2007), we mapped $q$ TIL 12 $2^{\text {W0120 }}, q N E I 12^{\text {W0120 }}$ and $q L E I 12^{\text {W0120. The QTLs }}$ were mapped between RM3739 and M10-3 (Fig. 4). According to the common molecular markers, the locations of $q T I L 12^{W 0120}, q N E I 12^{W 0120}$ and $q L E I 12^{W 0120}$ were integrated with the map of $q T I L 12^{C 9285}, q N E I 12^{C 9285}$ and $q L E I 12^{C 9285}$. The locations of $q T I L 12^{C 9285}, q N E I 12^{C 9285}$, and $q L E I 12^{C 9285}$ were the same as the locations of $q T I L 12^{\text {W0120 }}, q N E I 12^{\text {W0120 }}$ and $q L E I 12^{\text {W0120. }}$

\section{Discussion}

\section{Demonstration of the existence of QTLs in C9285}

We have detected QTLs that regulate internode elongation in deepwater rice using QTL analysis of the $\mathrm{F}_{2}$ population from the cross T65/C9285 (Hattori et al. 2007). To clone the QTLs, a demonstration of their existence and an evaluation of their effect are essential. In this study, we produced three NILs in a T65 genetic background: NIL-1 ${ }^{\text {C9285 }}$ possesses $q$ TIL1 $1^{C 9285}$, NIL-3 ${ }^{\text {C9285 }}$ possesses $q L E I 3^{C 9285}$ and NIL-12C9285 possesses qTIL12C9285, qNEI12C9285 and $q L E I 12^{C 9285}$. We compared the response to deep water of T65 and the three NILs. T65 slightly responded to deep water, but all NILs had larger scores than T65 (Fig. 2A-C). The results indicated that these QTLs functioned to enhance internode elongation in deep water. We demonstrated the accuracy of QTL analysis in the $\mathrm{F}_{2}$ population from the cross T65/C9285 (Hattori et al. 2007).

\section{Evaluation of QTL effects in C9285}

In previous QTL analysis, we detected five QTLs at three positions in C9285 (Fig. 1A; Hattori et al. 2007). Among these QTLs, three (qTIL12 ${ }^{C 9285}, q N E I 12^{C 9285}$ and $q L E I 12^{C 9285}$ ) locate on an overlapping region in the long arm of chromosome 12. Mapping in this study indicated that the QTLs are located at the same position: between RM6386 and RM235 (Fig. 4). Even though these three QTLs mapped to the same region, we still cannot clarify if they are independent and whether single QTL has effective TIL, NEI or LEI traits. It might be a single effective QTL regulates TIL, NEI and LEI rather than assuming three independent QTLs in this narrow region. In this study, we also mapped QTLs on chromosome 12 in W0120, qTIL12 $2^{\text {W0120 }}$, qNEI12 $2^{\text {W0120 }}$ and $q L E I 12^{W 0120}$. The results also indicated that the three QTLs cannot be dissected out and mapped to the same region between RM3739 and M10-3 (Fig. 4). Integrated QTL maps for chromosome 12 in W0120 and C9285 suggest that the location of the QTL in C9285 is involved in the location of the QTL in W0120. We suppose that the QTLs on chromosome 12 are a single and effective QTL for TIL, NEI and LEI traits and that QTL is common between the deepwater cultivar C9285 and the wild rice species (O. rufipogon, W0120).

qTIL1 ${ }^{\text {C9285 }}$ detected on chromosome 1 was identified by a single trait, TIL (Fig. 1A; Hattori et al. 2007). However, NIL-1 ${ }^{\text {C9285 }}$ possessing $q$ TIL1 $1^{\text {C9285 }}$ had higher TIL, NEI and LEI scores than those in T65. Namely, qTIL1 ${ }^{C 9285}$ has not

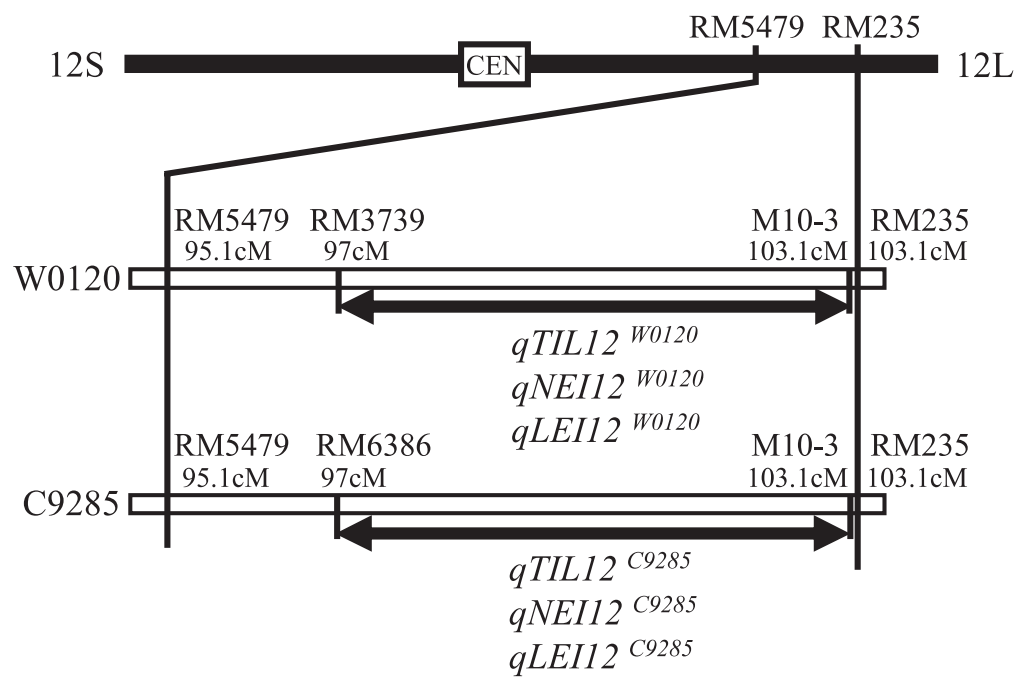

Fig. 4. Comparison of mapping region of QTLs on chromosome 12 in C9285 and W0120. Candidate region of QTLs, qTIL12 W0120, qNEI12 W0120 and $q L E I 12^{\text {W0120 }}$, in W0120 is between molecular markers RM3739 and M10-3. Candidate region of QTLs, $q$ TIL12 ${ }^{\text {C9285 }}, q^{\text {NEII2 }}{ }^{\text {C9285 }}$ and

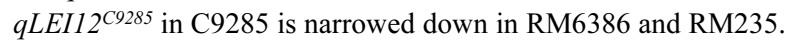


only a TIL trait, but also has NEI and LEI traits, even though the QTL only detected TIL traits. A QTL on chromosome 3, $q L E I 3^{C 9285}$, was identified as an LEI trait (Fig. 1A; Hattori et al. 2007), and NIL-3 ${ }^{\mathrm{C} 9285}$ possessing $q L E I 3^{C 9285}$ had higher TIL, NEI and LEI scores than those in T65, even though $q L E I 3^{C 9285}$ was only detected by a single trait, LEI. Hence, qTIL1 $1^{C 9285}$ and $q L E I 3^{C 9285}$ have the potential ability to enhance three traits (TIL, NEI and LEI) in the original C9285; however, QTL analysis using $\mathrm{F}_{2}$ populations could not demonstrate whether $q T I L 1^{C 9285}$ has NEI or LEI potential or whether $q L E I 3^{C 9285}$ has TIL or NEI potential. This may result from the single QTL analysis using small populations and because the QTL analysis in the $\mathrm{F}_{2}$ population cannot do repeat analysis, thus reducing the detection ability of the QTL. However, production of NIL allows us to detect effects that QTL analysis cannot detect and thus to evaluate the QTL effect exactly.

\section{Comparison of QTL effects}

The internode length of NIL-12 ${ }^{\mathrm{C} 9285}$ in deepwater conditions is larger than that of NIL-1 ${ }^{\mathrm{C} 9285}$ and NIL-3 ${ }^{\mathrm{C} 9285}$ (Fig. 2). This indicates that the QTL effect on the chromosome segment of chromosome 12 from C9285 is stronger than that in chromosome 1 in C9285 or chromosome 3 in C9285. NIL-12 ${ }^{\mathrm{C} 9285}$ may have three independent QTLs, $q_{T I L 1}{ }^{C 9285}, q L E I 3^{C 9285}$ and $q T I L 12^{C 9285}$, and we cannot exclude that the largest internode elongation effect of the chromosome 12 segment from C9285 comes form the additive effect of these three independent QTLs. However, as discussed above, according to mapping, we suggest that a single QTL regulating TIL, NEI and LEI traits exists in the region. In this context, the QTL on chromosome 12 is the most effective QTL for regulation of internode elongation in deepwater rice. QTL analysis based on static analysis is a powerful tool to look for QTLs regulating interesting target traits and offers QTLs positions, effects, and interactions. However, finding the exact effect of each QTL using NILs is more reliable. According to the production of NILs for deepwater characteristics, we can evaluate each QTL, and these lines are essential material for cloning the QTLs. The NILs could be used for pyramiding QTLs to understand the interactions of each QTL.

\section{Deepwater survival has been selected for breeding cultivars in flooded areas}

Among the detected QTLs from $\mathrm{F}_{2}$ populations between T65/C9285 and T65/W0120, the QTLs on chromosome 12 were common in C9285 and W0120 (Hattori et al. 2007). Mapping of QTLs in this study showed that the QTL positions in C9285 and W0120 completely overlapped (Fig.4), suggesting that the QTLs on chromosome 12 in C9285 (O. sativa ssp. indica) and in W0120 (O. rufipogon; perennial type) might be the same.

To elucidate the mechanism, evolution and domestication pathway of the deepwater function in rice, cloning the QTLs in chromosome 12 is essential. In this study, we pro- duced the NILs and also mapped the QTLs. This study has paved the way toward cloning the QTLs, and positional cloning of these QTLs is now in progress. Cloning the QTLs and functional analysis will elucidate the mechanism of internode elongation in response to deep water in deepwater rice.

\section{Acknowledgment}

We thank Professor Nori Kurata and Dr. Mitsugu Eiguchi for providing W0120 and C9285 seeds. The wild rice accessions used in this study were distributed from the National Institute of Genetics supported by the National Bioresource Project, MEXT, Japan. This work was supported by a grant from the Ministry of Agriculture, Forestry and Fisheries of Japan (MAFF; Green Technology Project QT2003) and Research Fellowships from the Japan Society for the Promotion of Science for Young Scientists.

\section{Literature Cited}

Ashikari,M. and M. Matsuoka (2006) Identification, isolation and pyramiding of quantitative trait loci for rice breeding. Trends Plant Sci. 11: 344-350.

Catling,D. (1992) Rice in deep water. International Rice Research Institute, Manila.

Chen, X., S.Temnykh, Y.Xu, Y.G.Cho and S.R.McCouch (1997) Development of a microsatellite framework map providing genome-wide coverage in rice (Oryza sativa L.). Theor. Appl. Genet. 95: 553-567.

Eiguchi,M., H.Y.Hirano, Y.Sano and H.Morishima (1993) Effect of water depth on internode elongation and floral induction in a deepwater-tolerant rice line carrying the $d w 3$ gene. Jpn. J. Breed. 43: 135-139.

Hamamura, K. and T.Kupkanchankul (1979) Inheritance of floating ability of rice. Jpn. J. Breed. 29: 211-216.

Hattori,Y., K.Miura, K. Asano, E.Yamamoto, H.Mori, H.Kitano, M.Matsuoka and M.Ashikari (2007) A major QTL confers rapid internode elongation in response to water rice in deepwater rice. Breed. Sci. 57: 305-314.

Inouye,J. (1983) Relation between elongation ability and internode elongation of floating rice under rising water conditions. Jpn. J. Trop. Agr. 27: 181-186.

Inouye,J. (1987) On floating rice and its ecological traits in Southeast Asia. Southeast Asia Studies 25: 51-61 (in Japanese).

Kende,H., E.van der Knaap and H.T.Cho (1998) Deepwater rice: A model plant to study stem elongation. Plant Physiol. 118: $1105-1110$

Konieczny, A. and F.M.Ausubel (1993) A procedure for mapping Arabidopsis mutations using co-dominant ecotype-specific PCR-based markers. Plant J. 4: 403-410.

McCouch,S.R., L.Teytelman, Y.Xu, K.B.Lobos, K.Clare, M.Walton, B.Fu, R.Maghirang, Z.Li, Y.Xing et al. (2002) Development and mapping of 2240 new SSR markers for rice (Oryza sativa L.). DNA Res. 9: 199-207.

Morishima,H., K.Hinata and H.I.Oka (1962) Floating ability and drought resistance in wild and cultivated species of rice. Indian J. Genet. 22: 1-11.

Nemoto,K., Y.Ukai, D.Q.Tang, Y.Kasai and M.Morita (2004) Inheritance of early elongation ability in floating rice revealed by diallel 
and QTL analyses. Theor. Appl. Genet. 109: 42-47.

Ramiah,K. and K.Ramaswami (1940) Floating habit in rice. Indian J. Agric. Sci. 11: 1-8.

Suge,H. (1987) Physiological genetics of internode elongation under submergence in floating rice. Jpn. J. Genet. 62: 69-80.

Tripathi,R.S. and M.J.BalakrishnaRao (1985) Inheritance studies of characters associated with floating habit and their linkage relationship in rice. Euphytica 34: 875-881.

Vergara,B.S., B.Jackson and S.K.DeDatta (1976) Deepwater rice and its response to deepwater stress. Climate and rice. International Rice Research Institute, Manila.
Vergara,B.S. and A.Mazaredo (1979) Using the new standard evaluation system to measure elongation ability. Proc 1978 Intl Deepwater Rice Workshop, International Rice Research Institute, Manila, pp. 139-142.

Ware,D.H., P.Jaiswal, J.Ni, I.V.Yap, X.Pan, K.Y.Clark, L.Teytelman, S.C.Schmidt, W.Zhao, K.Chang et al. (2002) Gramene, a tool for grass genomics. Plant Physiol. 130: 1606-1613.

Yano,M. (2001) Genetic and molecular dissection of naturally occurring variation. Curr. Opin. Plant Biol. 4: 130-135.

Yano,M. and T.Sasaki (1997) Genetic and molecular dissection of quantitative traits in rice. Plant Mol. Biol. 35: 145-153. 